\title{
A PROOF OF LIGGETT'S VERSION OF THE SUBADDITIVE ERGODIC THEOREM
}

\author{
SHLOMO LEVENTAL
}

(Communicated by Daniel W. Stroock)

\begin{abstract}
Using a method developed by Y. Katznelson and B. Weiss we give a proof of Liggett's improved version of the subadditive ergodic theorem.
\end{abstract}

1. Introduction. Lately (1985) an improved subadditive ergodic theorem due to Liggett has appeared [3]. To put things in context we quote Liggett's result:

Suppose $\left\{X_{m, n}\right\}$ is a collection of random variables indexed by integers satisfying $0 \leq m<n$. Assume

(i) $X_{0, n} \leq X_{0, m}+X_{m, n}$ whenever $0<m<n$.

(ii) (a) The joint distribution of $\left\{X_{m+1, m+k+1}: k \geq 1\right\}$ are the same as those of $\left\{X_{m, m+k}: k \geq 1\right\}$ for each $m \geq 0$.

(ii)(b) For each $k \geq 1,\left\{X_{n k,(n+1) k}: n \geq 1\right\}$ is a stationary process.

(iii) For each $n, E\left|X_{0, n}\right|<\infty$ and $E X_{0, n} \geq-c \cdot n$ where $c$ is a constant.

Then $\lim _{n} X_{0, n} / n$ exists a.s.

The reason for using the notation (ii)(a) and (ii)(b) is to help clarify the difference between Liggett's result and Kingman's original theorem. In Kingman's subadditive ergodic theorem instead of (ii)(a) and (ii)(b) there is the following condition: The joint distributions of $\left\{X_{m+1, n+1}: 0 \leq m<n\right\}$ are the same as those of $\left\{X_{m, n}: 0 \leq m<n\right\}$. In his paper Liggett explains the importance of this extension in applications.

The purpose of this note is to give a proof of Liggett's result. First we give a lemma which turns out to be the key ingredient in the proof. Then we express the theorem in the terminology of ergodic theory. From the lemma Birkhoff's ergodic theorem and Liggett's theorem both follow. The method of the proof of the lemma closely follows Katznelson and Weiss [1]. It turns out that this method is good enough to also prove Liggett's theorem.

The referee of this paper has pointed out that versions of Liggett's theorem have been proved recently. For example, see K. Schurger in Stochastic Process. Appl. 21 (1986).

\section{The proof.}

Setup. The quadruple $(\Omega, B, \mu, T)$ will denote the following objects: $\Omega$ is a space, $B$ is a $\sigma$-algebra, $\mu$ is a probability measure, and $T$ is a $B / B$ measurable function from $\Omega$ to $\Omega$.

DEFINITION. Let $S \subseteq B$ be a $\sigma$-algebra. $T$ will be called $S$ measure preserving (m.p.) if $\mu\left\{T^{-n}(A)\right\}=\mu\{A\}$ for every $A \in S$ and $n \geq 0$.

Received by the editors September 8, 1986.

1980 Mathematics Subject Classification (1985 Revision). Primary 60F15.

Key words and phrases. Subadditive ergodic theory. 
Note. If $f: \Omega \rightarrow \mathbf{R}$ is $S / B(\mathbf{R})$ measurable, $f \in L_{1}(\mu)$, and $T$ is $S$ m.p. then $E(f)=E\left(f\left(T^{n}\right)\right)$ for $n \geq 0$.

The next lemma is the key step in what follows.

LEMMA. Let $\left\{f_{n}\right\}$ be a sequence of $L^{1}(\mu)$ functions and assume that $T$ is $\sigma\left\{f_{k}: k \geq 1\right\}$ m.p. If

(i) $f_{n+m}(x) \leq f_{n}(x)+f_{m}\left(T^{n}(x)\right), n, m \geq 1$, and

(ii) for each $x \in \Omega$ there exists an integer $n(x)$ such that $f_{n}(x) \leq 0$, then $\lim \sup _{n} E f_{n} / n \leq 0$.

Proof. Take $n(x)=\min \left\{n \geq 1 \mid f_{n}(x) \leq 0\right\}$. Fix $\varepsilon>0$. Choose $N \geq 1$ large enough such that $\int_{A} f_{1}(x) d \mu \leq \varepsilon$ where $A=\{x: n(x)>N\}$.

Note that it follows from the definition of $A$ that $f_{1}(x) \geq 0$ for $x \in A$. Define

$$
\varphi(x)=\left\{\begin{array}{ll}
0, & x \notin A, \\
f_{1}(x), & x \in A,
\end{array} \quad m(x)= \begin{cases}n(x), & x \notin A, \\
1, & x \in A .\end{cases}\right.
$$

Note the following three facts:

(1) For $x \in \Omega, f_{m}(x) \leq \sum_{0 \leq i<m} \varphi\left(T^{i} x\right)$ because $\varphi \geq 0$ and $f_{m}(x) \leq 0$ if $x \notin A$,

(2) $m(x) \leq N$,

(3) $\varphi(x)$ is $\sigma\left\{f_{k}: k \geq 1\right\} / B(\mathbf{R})$-measurable.

As in [1] define inductively $m_{1}(x)=m(x)$ and $m_{k+1}(x)=m_{k}(x)+m\left(T^{m_{k}(x)}(x)\right)$. Take $L>N$. Let $k(x)=\max \left\{k: m_{k}(x) \leq L\right\}$. We now compute as follows, using (1):

$$
\begin{aligned}
& f_{m_{1}}(x) \leq \sum_{0 \leq j<m_{1}} \varphi\left(T^{j} x\right), \\
& f_{m_{2}-m_{1}}\left(T^{m_{1}}(x)\right) \leq \sum_{m_{1} \leq j<m_{2}} \varphi\left(T^{j} x\right), \\
& \vdots \\
& f_{m_{k}-m_{k-1}}\left(T^{m_{k}}(x)\right) \leq \sum_{m_{k-1} \leq j<m_{k}} \varphi\left(T^{j} x\right) .
\end{aligned}
$$

Using (i) repeatedly we get after adding the former inequalities

$$
f_{m_{k}}(x) \leq \sum_{0 \leq j<m_{k}} \varphi\left(T^{j} x\right)
$$

By using (i) we get

$$
f_{L}(x)-f_{m_{k}}(x) \leq \sum_{m_{k} \leq j \leq L-1} f_{1}\left(T^{j}(x)\right) .
$$

From the definition of $k$ we have $L-m_{k} \leq N$ so

$$
\sum_{m_{k} \leq j \leq L-1} f_{1}\left(T^{j}(x)\right) \leq \sum_{L-N \leq j \leq L}\left|f_{1}\left(T^{j}(x)\right)\right| .
$$

(*) can now be written as

$$
f_{L}(x) \leq \sum_{0 \leq j<L} \varphi\left(T^{j} x\right)+\sum_{L-N \leq j \leq L}\left|f_{1}\left(T^{j}(x)\right)\right| .
$$


If we integrate both sides (recall that $\varphi$ is $\sigma\left\{f_{k}: k \geq 1\right\}$-measurable) we get after dividing by $L$ :

$$
E\left(f_{L}\right) / L \leq E(\varphi)+(N / L) \cdot E\left(\left|f_{1}\right|\right) \leq \varepsilon+(N / L) \cdot E\left(\left|f_{1}\right|\right) .
$$

Letting $L \rightarrow \infty$ we get $\lim \sup _{n} E f_{n} / n \leq \varepsilon$. Since $\varepsilon$ is arbitrary the lemma is proved.

Denote for the rest of the paper $f=\liminf _{n}\left(f_{n} / n\right)$ and $F=\lim \sup _{n}\left(f_{n} / n\right)$.

We can modify the lemma to get the following result:

ClaIM. Let $\left\{f_{n}\right\}$ be a sequence of $L^{1}(\mu)$ functions and assume that $T$ is $\sigma\left\{f_{k}: k \geq 1\right\}$ m.p. If $f_{n+m}(x) \leq f_{n}(x)+f_{m}\left(T^{n}(x)\right), n, m \geq 1$, and $E(f)$ is defined then $E f \geq \lim \sup _{n} E f_{n} / n$.

PROOF OF THE CLAIM. W.l.o.g. we may assume $f^{+}=\max (f, 0) \in L^{1}(\mu)$ (if not then $f^{-} \in L^{1}(\mu)$ and $E(f)=\infty$ so there is nothing to prove). Fix $\varepsilon>0$ and $M>0$. Let us denote $f^{M}=\max \{f,-M\}$.

Note. $f^{M}$ is $T$ invariant. To see this use the relation $f_{n+m}(x) \leq f_{n}(x)+$ $f_{m}\left(T^{n}(x)\right)$. Fix $n$, divide by $m$ and let $m \rightarrow \infty$. We get $f(x) \leq f\left(T^{n}(x)\right)$ which in turn implies $f^{M}(x) \leq f^{M}\left(T^{n}(x)\right)$ but $E\left(f^{M}(x)\right)=E\left(f^{M}\left(T^{n}(x)\right)\right)<\infty$ since $f^{M} \in L^{1}(\mu)$ and is $\sigma\left(f_{k}: k \geq 1\right)$-measurable. So $f^{M}(x)=f^{M}\left(T^{n}(x)\right)$.

In order to use the lemma put $g_{n}=f_{n}-n \cdot\left(f^{M}+\varepsilon\right)$ and observe that the conditions of the lemma are satisfied for $T$ and $\left\{g_{n}\right\}$. So we get

$$
\underset{n}{\limsup } E\left[f_{n}-n \cdot\left(f^{M}+\varepsilon\right)\right] / n \leq 0
$$

which implies $E f^{M}+\varepsilon \geq \lim \sup _{n} E f_{n} / n$. But $\varepsilon$ and $M$ are arbitrary so the claim is proved.

REMARK. We can reformulate the claim as follows: Let $\left\{f_{n}\right\}$ be a sequence of $L^{1}(\mu)$ functions and assume that $T$ is $\sigma\left\{f_{k}: k \geq 1\right\}$ m.p. If $f_{n+m}(x) \geq f_{n}(x)+$ $f_{m}\left(T^{n}(x)\right), n, m \geq 1$, and $E(F)$ is defined then $E(F) \leq \liminf _{n} E f_{n} / n$.

COROLlARY. If $g \in L^{1}(\mu)$ and $T$ is $\sigma\left\{g\left(T^{n}\right): n \geq 1\right\}$ m.p. (in other words $\left\{g\left(T^{n}\right): n \geq 1\right\}$ is a stationary process) then

$$
\left.\left[\limsup _{n} \sum_{0 \leq i<n} g\left(T^{i}\right) / n\right)\right]^{+} \in L^{1}(\mu) \text { and } E\left(\limsup _{n} \sum_{0 \leq i<n} g\left(T^{i}\right) / n\right) \leq E(g) \text {. }
$$

(From reformulating the corollary in terms of lim inf together with the corollary as stated we get Birkhoff's ergodic theorem.)

PROOF. Note that $\lim \sup _{n} \sum_{0 \leq i<n} g\left(T^{i}\right) / n \leq \limsup \operatorname{su}_{n} \sum_{0 \leq i<n} g^{+}\left(T^{i}\right) / n$. Now we use the remark: Put $f_{n}=\sum_{0 \leq i<n} g^{+}\left(T^{i}\right)$. So we get

$$
E\left(\limsup _{n} \sum_{0 \leq i<n} g^{+}\left(T^{i}\right) / n\right) \leq E\left(g^{+}\right)<\infty .
$$

This means that $\left.\left[\lim \sup _{n} \sum_{0 \leq i<n} g\left(T^{i}\right) / n\right)\right]^{+} \in L^{1}(\mu)$ so we may use again the remark for $f_{n}=\sum_{0 \leq i<n} g\left(T^{i}\right)$ and get the result. 
And now we can prove the main theorem:

THEOREM. Let $\left\{f_{n}\right\}$ be a sequence of $L^{1}(\mu)$ functions. Assume

(i) $f_{n+m}(x) \leq f_{n}(x)+f_{m}\left(T^{n}(x)\right), n, m \geq 1$.

(ii)(a) $T$ is $\sigma\left\{f_{k}: k \geq 1\right\}$ m.p.

(ii)(b) For each $k, T^{k}$ is $\sigma\left\{f_{k}\left(T^{k \cdot n}\right): n \geq 1\right\}$ m.p. (in other words: For each $k$, $\left\{f_{k}\left(T^{k \cdot n}\right): n \geq 1\right\}$ is a stationary process).

(iii) For each $n, E\left(f_{n}\right) \geq-c \cdot n$ for a constant $c$.

Then $f_{n} / n$ converges a.s. $(\mu)$.

PROOF.

FIRST STEP. $E\left(f_{n}\right) / n$ converges to $\gamma$ (say) and $\gamma=\inf _{n \geq 1}\left\{E\left(f_{n}\right) / n\right\}$.

ProOF. $E\left(f_{n+m}\right) \leq E\left(f_{n}\right)+E\left(f_{m}\right)$ because of (i) and (ii)(a). Now we get the conclusion by a well-known fact about subadditive sequences of numbers (e.g. Liggett [3, p. 1281]). Note that $-c \leq \gamma<\infty$.

SECOND STEP. $F^{+} \in L^{1}(\mu)$ and $E(f) \leq \gamma$.

PrOOF. Fix $k \geq 1$. Note that $F=\lim \sup _{n}\left\{f_{k \cdot n} /(k \cdot n)\right\}$ since $f_{k \cdot n+j} \leq$ $f_{k \cdot n}+f_{j}\left(T^{k \cdot n}\right)$ and, when $j$ is fixed and $n \rightarrow \infty, f_{j}\left(T^{k \cdot n}\right) / n \rightarrow 0$ a.s. by BorelCantelli.

By using (i) we get $f_{k \cdot n}(x) \leq \sum_{0 \leq i \leq n-1} f_{k}\left(T^{k \cdot i}\right)$. So

$$
F^{+} \leq\left[\limsup \sum_{0 \leq i \leq n-1} f_{k}\left(T^{k \cdot i}\right) /(k \cdot n)\right]^{+}
$$

and

$$
F \leq \limsup \sum_{0 \leq i \leq n-1} f_{k}\left(T^{k \cdot i}\right) /(k \cdot n) .
$$

Now we use the corollary for $g=f_{k} / k$ and we get from the first inequality that $F^{+} \in L^{1}(\mu)$ and from the second that $E(F) \leq E\left(f_{k} / k\right)$ which implies that $E(F) \leq$ $\gamma$ since $k$ is arbitrary.

THIRD STEP. $E(f) \geq \gamma$ where $f=\liminf _{n}\left(f_{n} / n\right)$.

PROOF. $f^{+} \leq F^{+}$. Since $F^{+} \in L^{1}(\mu)$ as we saw in the second step we conclude that $f^{+} \in L^{1}(\mu)$ so $E(f)$ is defined and now the third step follows from the claim.

We have proved that $E(F) \leq \gamma \leq E(f)$ but $F \geq f$ so we must have $F=f$ a.s. $\mu$ and the proof of the theorem is finished.

REMARK. This theorem is equivalent to Liggett's Theorem 1.10 in [3] with the following identifications:

$\Omega \in \mathbf{R}^{H}$ where $H=\{(m, n): 0 \leq m<n\}$ and $\mathbf{R}$ denotes the real numbers.

$T$ is a transformation on $\Omega$ defined by $T: x \rightarrow y$ where $y(m, n)=x(m+1, n+1)$.

$f_{m}=X_{0, m}$.

$f_{n-m}\left(T^{m}\right)=X_{m, n}$.

Note that assumption (1.8) in Liggett is, in our terminology, that $T$ is measure preserving in the sense stated in our theorem, namely: $\mu\left(T^{-i}(A)\right)=\mu(A)$ for each $A \in \sigma\left\{f_{n}\right\}$ and $i=1,2, \ldots$; while in Kingman's result $T$ has to be measure preserving in the following sense: $\mu\left(T^{-1}(A)\right)=\mu(A)$ for each $A \in B$ where $B$ is the minimal $\sigma$-algebra generated by $\left\{X_{l}: l \in H\right\}$. 


\section{BIBLIOGRAPHY}

1. Y. Katznelson and B. Weiss, A simple proof of some ergodic theorems, Israel J. Math. 42 (1982), 291-296.

2. J. F. C. Kingman, Subadditive ergodic theory, Ann. Probab. 1 (1973), 883-909.

3. T. M. Liggett, An improved subadditive ergodic theorem, Ann. Probab. 13 (1985), 1279-1285.

Department of Probability and Statistics, Michigan State University, EAST LANSING, MICHIGAN 48824 